\title{
Immunity of coherent stroboscopic processing system of ultra wideband signals during synchronous mode
}

\author{
Oleg Pak ${ }^{1, *}$ and Vladimir Zakharchenko ${ }^{2}$ \\ ${ }^{1}$ LLC “Averia Electronics”, 400010, 39 Kachintsev St., Volgograd, Russia \\ ${ }^{2}$ Volgograd State University, Radio Physics Department, 400062, 100 Universitetskii Ave., \\ Volgograd, Russia
}

\begin{abstract}
Analyze of the radio pulse stroboscopic schema work in "synchronous" mode was made at clock interval synchronization by the second harmonic of intermediate frequency. It's introduced relationships describing the input radio signal's amplitude relationship during the phase modulation transforming in time during work the converter in synchronous mode. The results of semi natural modeling of the radio pulse gating system qualitative agreement with theoretical calculations.
\end{abstract}

\section{Introduction}

Radio technical systems using signals of nanosecond duration have some advantages: a high resolution range can be reached, stealth, electromagnetic compatibility in conditions of working with narrow band radar systems in the same time etc. [1,2]. The probing signal of the radiolocation station is a periodic series of nanosecond duration microwave radio pulses and a processing this kind of signals usually is doing by using stroboscopic methods [3].

\section{Radio pulse stroboscopic converter. Synchronous mode}

Mathematical model of radio pulse gating system is shown in the Fig.1.

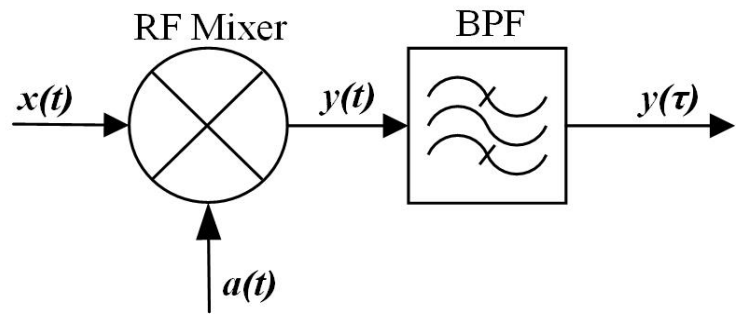

Fig. 1. It's a linear mathematical model of radio pulse stroboscopic converter: the RF Mixer - radio frequency mixer, the BPF - band pass filter.

\footnotetext{
* Corresponding author: oleg.pak86@gmail.com
} 
The stroboscopic converter of a periodical series of coherent radio pulses $x(t)$ works like a super heterodyne receiver and the series of strobe pulses $a(t)$ is using as a local oscillator signal $[4,5]$. Mathematical models of the input signal and the strobe signal are:

$$
\begin{aligned}
& x(t)=\sum_{k=0}^{N} \mathrm{X}\left(t-k T-t_{0}\right) \cos \left[\omega_{0} t+\theta(t-k T-\tau)\right] ; \\
& a(t)=\sum_{k=0}^{N} A\left(t-k T_{1}-t_{0}\right) \cos \left[\omega_{1} t+\varphi\left(t-k T_{1}-\tau\right)\right] .
\end{aligned}
$$

In this relationship $X(t), A(t)$ are complex envelopes, $\omega_{0}, \omega_{1}$ - carrier wave frequencies; $\theta(t), \varphi(t)$ - intrapulse phase modulation of the input $x(t)$ signal and strobe radio pulse $a(t)$ respectively. The delay $t_{0}$ describes a time offset between these radio pulse and radio strobe envelopes relative to a high frequency filling.

$\omega_{0}-\omega_{1}=\Omega\left(\Omega<<\omega_{0}, \omega_{1}\right)-$ low intermediate frequency; $T$ - input signal repetition period;

$T_{1}=T+\Delta T ; \Delta T=T / N$ - sampling step; $N$ - spectral transform coefficient $(N>>1)$.

Take into account $N \rightarrow \infty$ (it usually is $N: 10^{4} \div 10^{6}$ in real system), an output signal of RF mixer can be asymptotically present in the following form:

$$
y(t) \cong \frac{\widetilde{A}}{4 T} \sum_{n} X\left(\frac{t}{N}\right) \cos \left\{\left[\left(\omega_{n}+\Omega\right) t_{0}+\theta\left(\frac{t}{N}\right)-\varphi_{0}\right]+\cos \left[\left(\omega_{n}-\Omega\right) t_{0}-\theta\left(\frac{t}{N}\right)+\varphi_{0}\right]\right\} \cdot
$$

The $\widetilde{A}$ is a square of the stroboscopic radio pulse envelope, $\varphi_{0}$ - an average phase of that radio pulse, $\omega_{n}=2 \pi n / T_{1}$ - the frequency of the harmonic number $n$ of the stroboscopic pulses repetition frequency $(n=1,2,3 \ldots)$.

Filtering by the system's band pass filter close spectral components with $\Omega$ and $\omega_{1}-\Omega$ frequencies (Fig. 2) and synchronizing the repetition frequency of the input signal by the second harmonics of the intermediate $\Omega$ frequency (this is a synchronous mode), the expression (1) can be represent in the form:

$$
\widetilde{y}(t) \cong \frac{\widetilde{A}}{2 T} \times\left(\frac{t}{N}\right) \cos \left[\theta\left(\frac{t}{N}\right)+\Omega t_{0}-\varphi_{0}\right] \cos \left(\frac{\pi t}{T_{1}}\right) .
$$

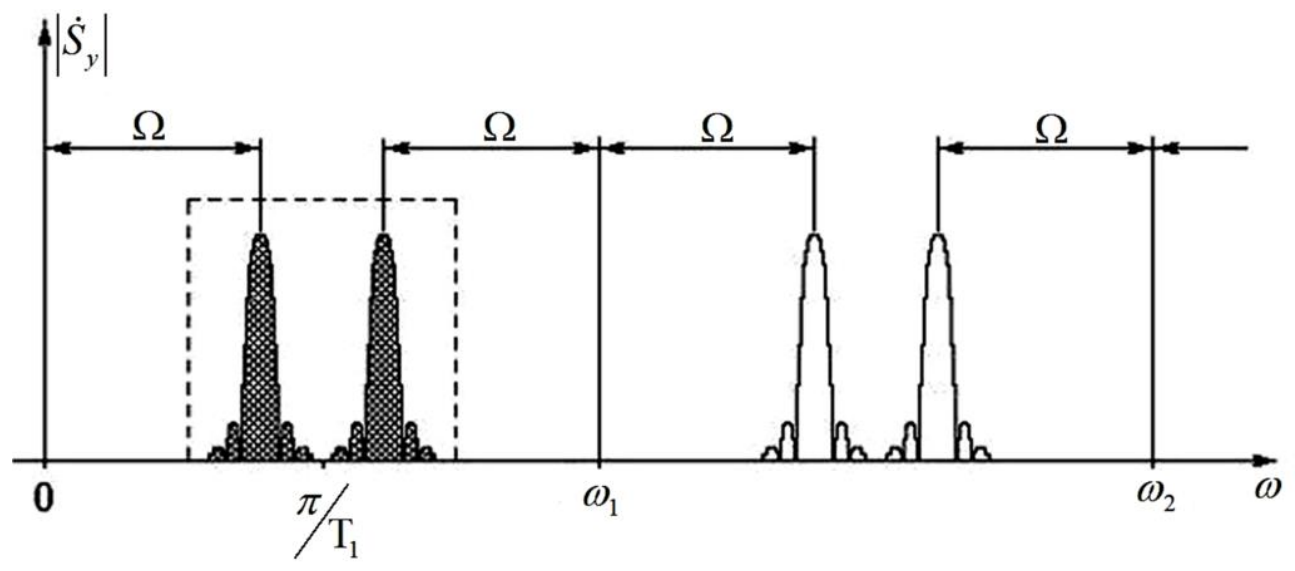

Fig. 2. It's shown a synchronization of spectral components with $\Omega$ and $\omega_{1}-\Omega$ frequencies. 
We can conclude from the equation (2) that it's possible to compensate a transforming signal's amplitude at points $t_{k}$ by matching of the phase $\varphi_{0}$ else the delay $t_{0}$, $\theta\left(t_{k} / N\right)=\pi / 2-\Omega t_{0}+\varphi_{0}$

The synchronous mode of the radio pulse stroboscopic converter was experimentally verified using the designed natural model of the radio pulse gating system, whose blockschema is shown in the figure $3[6]$.

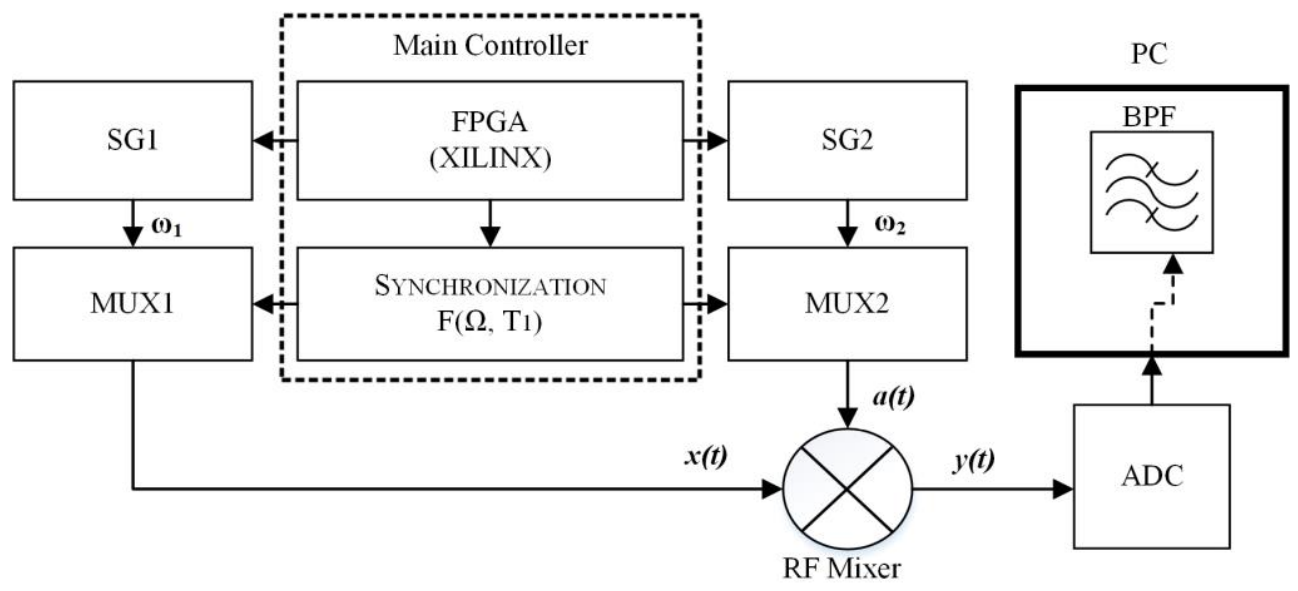

Fig. 3. This is a block-schema of the radio pulse gating system to do semi natural tests.

The direct digital frequency synthesizers SG1 and SG2 are generating harmonic signals with $\omega_{1}=10.0 \mathrm{M \Gamma ц} \mathrm{and} \omega_{2}=10.010 \mathrm{MГц} \mathrm{frequencies} \mathrm{respectively.} \mathrm{The} \mathrm{manager} \mathrm{schema}$ (Main Controller) is based on field-programmable gate array (FPGA). The schema manages to these digital radio frequency multiplexor (MUX1 and MUX2) and it producing a coherent series of an input and a stroboscopic radio pulses with $\tau_{x}=30$ us и $\tau_{a}=0.5$ us duration respectively. The spectral transformation coefficient of the useful signal's envelope is about $\mathrm{N}=5000$ at sampling step is $\Delta \mathrm{T}=10 \mathrm{~ns}$.

The synchronization module presents a logical unit in the FPGA core. The module is synchronizing the repetition frequency of the probing radio pulses by the second harmonic of the intermediate frequency $\Omega$ of the signal transforming in time.

The radio frequency mixer (RF Mixer) is an intermediate frequency correlator with built-in an analog low pass filter (LPF) with cutoff frequency is about $20 \mathrm{kHz}$. The intermediate frequency signal $y(t)$ pass through the analog-to-digital converter (ADC) module after that the digital data transmit to a personal computer for a processing purpose.

The narrow band bandpass filter of the radio pulse gating system is a digital transversal filter with pass band is about $50 \mathrm{~Hz}$ at $-3 \mathrm{~dB}$ level and a central frequency $\Omega=\omega_{2}-\omega_{1}=10 \mathrm{kHz}$. We used the Blackman window as a weight function to calculate an impulse response coefficient of the BPF. The Blackman window has the following characteristics: stopband attenuation is $\sim 57 \mathrm{~dB}$, ripple $\sim 0.002 \mathrm{~dB}$. The bandpass filter's impulse response function has $\sim 11000$ coefficients.

By selecting a repetition frequency of probing pulses equal $F_{n}=2 \Omega=20 \mathrm{kHz}$, the radio pulse stroboscopic converter will start working in synchronous mode. We can observe a superposition of spectral components at $\Omega$ and $F_{n}-\Omega$ frequencies of the signal transformed in time. 
As we can obtain from modeling results an envelope of the transformed signal might be distorted in phase sensitive mode. This is due to a parasitic phase modulation which is a specific disturbance in synchronous mode. This disturbance is due to differences between a repetition frequency of the input signal and strobe pulses too. It's possible to compensate the disturbance by swapping the synchronization sources of the signal under investigation and strobe radio pulses.

\section{Conclusion}

Theoretical analysis a possibility of work a radio pulse stroboscopic system in phase sensitive (synchronous) mode was made. It was shown the synchronous mode makes it possible to transform a signal's phase structure to amplitude modulation. And it also allows to make phase measurements by using a simple equipment.

It's proposed a compensation way of the specific parasitic phase modulation of the useful signal in synchronous mode.

The phase sensitive operation mode of the coherent radio pulse gating system allows us to achieve an increasing a signal-noise ratio about $\sim 3 \mathrm{~dB}$, thanks to a pairwise superposition of spectral components at $\Omega$ and $\omega_{1}-\Omega$ frequencies.

The results of a semi-natural simulation of the operation of a radio pulse stroboscopic converter in the synchronous mode are in qualitative agreement with the theoretical analysis.

This work is based on materials of research conducted with financial support of the Russian Foundation for Basic Research and the Volgograd Oblast Administration as part of the scientific project № 19-4340005-р_поволжье_а.

\section{References}

1. V.G. Radzievskiy, P.A. Trifonov, Obrabotka sverhshirokopolosnyh signalov I pomeh (in Russian, Moscow: Radiotehnika, 288 p.) (2009)

2. V.D. Zakharchenko, I.G. Kovalenko, O.V. Pak, Estimate of sizes of small asteroids (cosmic bodies) by the method of stroboscopic radiolocation, Earth and Planetary Astrophysics (Dec. 2014), doi: 10.1016/j.actaastro.2014.12.006 (2014)

3. A.I. Naydenov, Transformatsiya spectra nanosecundnyh impulsov (in Russian, Moscow: Soviet radio, 180 p.) (1973)

4. V.D. Zackharchenko, O.V. Pak, Potential of radar pulse strobing scheme use in phasesensitive operation mode, Radiation and Scattering of Electromagnetic Waves (RSEMW-2017), doi:10.1109/RSEMW.2017.8103641 (2017)

5. V.D. Zakharchenko, I.G. Kovalenko, O.V. Pak, V.Yu. Ryjkov, Fundamental restrictions on the coherence of probing signals in the task of achieving maximum resolution and range in the stroboscopic location of asteroids (in Russian), Cosmic research, vol. 56, no. 3, pp. 209-217 (2018)

6. V.D. Zackharchenko, O.V. Pak, Modelirovanie radioimpulsnogo stroboscopicheskogo preobrazovatelya (in Russian, Metody I ustroystva formirovaniya I obrabotki signalov v informatsionnyh sistemah, Ryazan, 5-8 pp.) (2010) 